\title{
ADIÇÃO DE ÓLEO DE COPAÍBA (Copaifera langsdorffii) E SUCUPIRA (Pterodon emarginatus) NA ALIMENTAÇÃO DE POEDEIRAS: QUALIDADE FÍSICA DE OVOS ARMAZENADOS EM DIFERENTES TEMPERATURAS
}

\author{
DIETARY SUPPLEMENTATION WITH PLANT OILS FROM COPAÍBA \\ (Copaifera langsdorffii) AND SUCUPIRA (Pterodon emarginatus): QUALITY OF \\ PHYSICAL ASPECTS OF EGGS STORED UNDER DIFFERENT \\ TEMPERATURES
}

\author{
Geovana Rocha de Oliveira ${ }^{1 *}$ \\ Cristiane Bovi de Lima ${ }^{1}$ \\ Luiza Maria Carvalho Soares Ribeiro ${ }^{1}$ \\ Marcos Barcellos Café ${ }^{2}$ \\ Janaina da Silva Moreira² \\ Eduardo Miranda de Oliveira² \\ Aline Mondini Calil Racanicci ${ }^{1}$ \\ 1Universidade de Brasília, Brasília, DF, Brasil \\ 2Universidade Federal de Goiás, Goiânia, GO, Brasil \\ *Autora para correspondência - geovanarocharural@yahoo.com.br
}

\section{Resumo}

O objetivo deste trabalho foi avaliar os efeitos da adição de óleos vegetais à alimentação de poedeiras semipesadas na qualidade física de ovos armazenados sob refrigeração (R) e em temperatura ambiente (TA). As aves receberam ração formulada à base de milho e farelo de soja com a suplementação de níveis crescentes de óleo de Copaifera langsdorffii (CP-0,03; 0,06 e 0,09\%) ou Pterodon emarginatus (SC-0,03 e 0,06\%) mais um controle negativo $(\mathrm{CN})$. Periodicamente, foram analisados três ovos por tratamento para Unidade Haugh (UH), índice de gema (IG), cor, pH (gema e albúmen) e porcentagens de casca (PC), gema (PG) e albúmen (PA). Para análise estatística dos resultados, foi adotado um modelo misto (SAS 9.3) e as médias foram comparadas pelo teste de Tukey. A inclusão de até $\mathrm{CP} 0,06 \% \mathrm{CP}$ ou $\mathrm{SC} 0,03 \%$ preservou $(\mathrm{P}<0,05)$ os valores de $\mathrm{UH}$ nos ovos em TA, equivalente aos tratamentos sob R até 14 dias; além disso, a adição dos óleos controlou a elevação do $\mathrm{pH}$ das gemas. Concluiu-se que os óleos vegetais suplementados nas dietas de poedeiras influenciaram positivamente na manutenção da qualidade do albúmen e do $\mathrm{pH}$ das gemas dos ovos em temperatura ambiente, contudo nenhum efeito adicional foi verificado para os ovos mantidos sob refrigeração.

Palavras-chave: antioxidantes naturais; ovos armazenados; poedeiras semipesadas; qualidade física.

\begin{abstract}
The aim of this study was to evaluate the effects of the dietary supplementation of laying hens with natural oils on the quality of fresh eggs stored under refrigeration (R) and room temperature (RT). Hens were fed balanced corn-soybean diets supplemented with increasing levels of Copaifera langsdorffii (CP-0.03; 0.06 and 0.09\%) or Pterodon emarginatus (SC-0.03 and 0.06\%) oilresins plus a negative control $(\mathrm{CN})$. Periodically, three eggs per treatments were evaluated for haugh unit (HU);
\end{abstract}


yolk index (YI); yolk color ( $\left.\mathrm{L}^{*}, \mathrm{a}^{*} \mathrm{e} \mathrm{b}^{*}\right)$; yolk and albumen $\mathrm{pH}$ and percentages of shell (PS); albumen (PA) and yolk (PY). Statistical analysis was performed using a mixed model (SAS 9.3) and Tukey test. The supplementation of $0.06 \% \mathrm{CP}$ and $0.03 \% \mathrm{SC}$ preserved $(\mathrm{p}<0.05) \mathrm{HU}$ in eggs stored in RT during 14 days, similarly to results found in R. In addition, dietary supplementation of natural oils helped to control $\mathrm{pH}$ levels in yolks during RT storage. In conclusion, the dietary supplementation of copaiba and sucupira showed positive effects on albumen quality and yolk $\mathrm{pH}$ for eggs stored at room temperature, however, no additional effect was found for eggs stored chilled.

Keywords: egg quality; laying hens; natural antioxidants; storage assay.

Recebido em: 27 de maio de 2016.

Aceito em: 26 de março de 2018.

\section{Introdução}

No cerrado brasileiro, em decorrência da grande variedade de espécies vegetais, caracterizadas pela presença de metabólicos secundários ${ }^{(1)}$, pesquisas com utilização de plantas como aditivos naturais são incentivadas, pois algumas plantas apresentam elevado potencial de compostos bioativos a serem explorados, em virtude da variedade de metabólicos primários e secundários produzidos ${ }^{(2)}$.

A Copaifera langsdorffii é uma árvore de grande porte, conhecida como copaíba, pau-d'óleo ou copaibeira. O gênero Copaifera possui componentes de interesse econômico e fitoterápico de conhecimento da medicina popular e científica, os quais podem ser avaliados em extratos de folhas, flores, frutos e óleo resina ${ }^{(3)}$. O conhecimento dos compostos e de sua aplicação na medicina popular incentivou o desenvolvimento de pesquisas para avaliar a atividade química e biológica dessa planta, entre elas as atividades anti-inflamatória ${ }^{(4)}$, antimicrobiana, cicatrizante ${ }^{(5)}$, antiulcerativa ${ }^{(3)}$ e antioxidante $^{(6)}$.

A espécie Pterodon emarginatus é uma planta arbórea, que alcança de 5 a 10 metros de altura, sendo popularmente conhecida como sucupira branca, faveiro e fava de sucupira ${ }^{(7)}$. Estudos científicos têm comprovado seus efeitos quanto à atividade cicatrizante, antiulcerogênica, anti-inflamatória ${ }^{(8)}$, $\operatorname{antimicrobiana}^{(9)}$ cercaricida $^{(10)}$ e antioxidante $^{(11,12)}$.

O uso de aditivos antioxidantes é uma prática amplamente empregada ${ }^{(13)}$, na qual a produção animal e a tecnologia de alimentos têm investido em pesquisas que avaliam, tanto os antioxidantes sintéticos quanto os naturais, em relação aos seus efeitos sobre o tempo de prateleira e qualidade nutricional dos alimentos ${ }^{(14,15)}$. A temperatura de armazenamento, também está entre os fatores que vem sendo empregado para manter a qualidade físico-química e nutricional dos alimentos. Estudos revelam que o armazenamento sob refrigeração pode minimizar os efeitos negativos que o tempo de armazenamento causa na qualidade dos ovos ${ }^{(16)}$.

A suplementação de aditivos herbais na alimentação de poedeiras vem sendo analisada, especialmente quanto aos seus efeitos nos aspectos de qualidade física dos ovos ${ }^{(17,18)}$. Porém, há carência de estudos avaliando a ação desses compostos vegetais sobre a qualidade físico-química de ovos armazenados, principalmente em temperatura ambiente, já que, no mercado interno brasileiro, $92 \%$ dos ovos são comercializados in natura, sem qualquer refrigeração ${ }^{(19)}$. Dessa forma, a legislação vigente apenas 
recomenda que os ovos em casca sejam armazenados entre 4 a $12{ }^{\circ} \mathrm{C}$, com controle de umidade relativa do ar, por no máximo 30 dias $^{(20)}$. Assim, o objetivo neste estudo é avaliar a adição de óleos vegetais à ração de poedeiras sobre a qualidade física de ovos armazenados em temperatura ambiente e refrigerados.

\section{Material e Métodos}

O óleo bruto de Pterodon emarginatus (sucupira) foi obtido por prensagem a frio das favas, adquiridas em comércio local. O óleo resina de Copaifera langsdorffii (copaíba), extraído do seio lenhoso do caule, foi comprado de cooperativas extrativistas e padronizado. Nos óleos de sucupira e copaíba foi quantificado o teor de $\beta$-cariofileno pelo método covalidado utilizando um cromatógrafo líquido de alta eficiência (CLAE) Waters ${ }^{\circledR}$ (Massachussets, USA), obtendo concentrações de 21,31\% no óleo resina de copaíba e 7,36\% no óleo de sucupira.

Neste estudo foram utilizadas 184 aves da linhagem ISA-Brown alojadas aos pares em gaiolas de $25 \times 40 \times 45 \mathrm{~cm}$, em galpão experimental de postura, distribuídas em seis tratamentos e sete repetições de quatro aves cada, em um delineamento inteiramente casualizado. As gaiolas eram equipadas com comedouros tipo calha em aço galvanizado e bebedouros tipo "nipple".

Em toda a fase de produção, as poedeiras receberam dieta formulada à base de milho e farelo de soja, segundo as recomendações de Rostagno $(2011)^{(21)}$ para poedeiras semipesadas em fase de produção. Os tratamentos foram compostos da ração basal (Tabela 1) com suplementação de três níveis de óleo de copaíba $(0,03 ; 0,06$ e $0,09 \%)$ e dois níveis de óleo de sucupira $(0,03$ e $0,06 \%)$ em substituição ao amido e mais um tratamento controle negativo com $0,5 \%$ de amido.

A coleta dos ovos in natura foi realizada às 37 semanas de idade, durante 3 (três) dias consecutivos, totalizando 667 ovos, que foram armazenados em geladeira a $10{ }^{\circ} \mathrm{C}$ até o início do ensaio de armazenamento.

Imediatamente após a coleta, os ovos foram distribuídos em um ensaio de armazenamento sob temperatura ambiente (TA) por 30 dias e sob refrigeração (R) em câmara fria $\left(4^{\circ} \mathrm{C}\right)$ por 60 dias. Durante o período experimental, foi aferida a temperatura ambiente diariamente, pela manhã e à tarde, sendo registradas temperaturas médias mínimas de 20 a $22{ }^{\circ} \mathrm{C}$ e máximas de 28 a $34{ }^{\circ} \mathrm{C}$ durante o período. As análises foram realizadas em 3 (três) ovos por tratamento, correspondentes aos dias 1, 2 e 3 de coleta. Periodicamente, as análises foram realizadas com 0, 7, 14, 21, 30, 35, 42, 49, 56 e 60 dias de armazenamento.

Os parâmetros observados foram unidade Haugh (UH), índice de gema (IG), pH da gema, pH do albúmen, cor da gema, porcentagem de casca (PC), gema (PG) e albúmen (PA). O valor de UH foi obtido utilizando a fórmula descrita por Pardi (1977), sendo: $\mathrm{UH}=100 \log \left(\mathrm{H}+7,57-1,7 \mathrm{~W}{ }^{0,37}\right)$, em que: $\mathrm{H}=$ altura do albúmen ( $\mathrm{mm})$ e $\mathrm{W}=$ peso do ovo $(\mathrm{g})$. O IG foi calculado de acordo com Funk (1973) utilizando a seguinte equação: $\mathrm{IG}=\mathrm{h} / \mathrm{d}$, em que: $\mathrm{h}=$ altura da gema $(\mathrm{mm})$ e $\mathrm{d}=$ diâmetro da gema (mm). O pH da gema e do albúmen foi determinado em uma leitura efetuada em "pool" de três ovos por dia de avaliação, utilizando-se pHmetro portátil da marca Testo, modelo T 205. A cor das gemas foi avaliada em triplicata, em três ovos por tratamento, utilizando colorímetro portátil da marca 
Konica Minolta, modelo Chroma Meter CR-400 operando no sistema CIELab (L*, a* e b*). Para o cálculo de PC, PG e PA foi aferido o peso do ovo e da gema, e o peso do albúmen foi determinado pela diferença do peso do ovo em relação ao peso da gema mais a casca. Os percentuais de casca, gema e albúmen foram calculados a partir dos seus respectivos pesos, divididos pelo peso do ovo e multiplicado por 100 .

Tabela 1. Composição da ração experimental basal para a fase de produção de poedeiras semipesadas

\begin{tabular}{|c|c|}
\hline Ingredientes (\%) & Fase de produção \\
\hline Milho & 65,82 \\
\hline Farelo de soja $45 \%$ & 20,79 \\
\hline Calcário calcítico (pedrisco) & 9,16 \\
\hline Óleo de soja & 1,50 \\
\hline Fosfato bicálcico & 0,99 \\
\hline Amido & 0,50 \\
\hline Sal comum & 0,45 \\
\hline DL-metionina & 0,25 \\
\hline L-lisina HCL & 0,25 \\
\hline L-treonina & 0,14 \\
\hline Suplemento vitamínico ${ }^{1}$ & 0,10 \\
\hline Suplemento mineral ${ }^{2}$ & 0,05 \\
\hline \multicolumn{2}{|l|}{ Composição química calculada ${ }^{3}$} \\
\hline \multicolumn{2}{|l|}{ Nutrientes } \\
\hline Energia metabolizável (Kcal/Kg) & 2.900 \\
\hline Proteína bruta (\%) & 15,60 \\
\hline Lisina digestível (\%) & 0,85 \\
\hline Metionina + cistina digestível (\%) & 0,69 \\
\hline Treonina digestivel (\%) & 0,63 \\
\hline Cálcio (\%) & 3,85 \\
\hline Fósforo disponivel (\%) & 0,28 \\
\hline Sódio (\%) & 0,21 \\
\hline \multicolumn{2}{|c|}{$\begin{array}{l}\text { Suplemento vitaminico - niveis de garantia por quilograma de produto: Vitamina A } 8.000 \text { UI, Vitamina E } 15.000 \mathrm{mg} \text {, } \\
\text { Vitamina D3 } 2.300 \mathrm{UI} \text {, Vitamina K3 } 1.000 \mathrm{mg} \text {, Vitamina B1 } 200 \mathrm{mg} \text {, Vitamina B2 } 3.000 \mathrm{mg} \text {, Vitamina B6 } 1.700 \mathrm{mg} \text {, } \\
\text { Vitamina B12 } 10.000 \mathrm{mcg} \text {, Niacina } 20.000 \mathrm{mg} \text {, Ácido fólico } 500 \mathrm{mg} \text {, biotina } 15,00 \mathrm{mg} \text {. }{ }^{2} \text { Suplemento mineral - niveis } \\
\text { de garantia por quilograma de produto: Manganês } 120.000 \mathrm{mg}, \text { Zinco } 120.000 \mathrm{mg} \text {, Ferro } 60.000 \mathrm{mg} \text {, Cobre } 18.000 \mathrm{mg} \text {, } \\
\text { Iodo } 2.000 \mathrm{mg} \text {, Cálcio } 9.600 \mathrm{mg} \text {. " Valores calculados baseados em Rostagno et al. }{ }^{(21)} \text {. }\end{array}$} \\
\hline
\end{tabular}

No ensaio de armazenamento foi utilizado um delineamento inteiramente casualizado com estrutura de tratamentos fatorial $2 \times 3 \times 2$ (duas plantas, três níveis, duas temperaturas de armazenamento) com um tratamento controle negativo adicional. $\mathrm{O}$ período de armazenamento foi considerado como um fator longitudinal, variando de 5 tempos ( 0 a 30 dias) sob R e em TA, até 9 tempos ( 0 a 60 dias) sob R.

Os dados foram comparados utilizando um modelo misto, com efeito fixo para os tratamentos e aleatório para os períodos de armazenamento, utilizando-se o procedimento PROC MIXED do software Statistical Analisys System (SAS 9.3) ${ }^{(22)}$. As médias foram comparadas pelo teste de Tukey em 5\% de nível de significância. 


\section{Resultados e Discussão}

Os valores médios de $\mathrm{UH}, \mathrm{IG}, \mathrm{PC}, \mathrm{PG}, \mathrm{PA}, \mathrm{a}^{*} \mathrm{e} \mathrm{b}^{*}$ não diferiram $(\mathrm{p}<0,05)$ entre os tratamentos nos ovos armazenados por até 30 dias em TA (Tabela 2). Em estudos semelhantes ${ }^{(18)}$, a adição de 100 e $200 \mathrm{mg} / \mathrm{kg}$ de óleo essencial de orégano na dieta de poedeiras não influenciou significativamente os parâmetros de UH, diâmetro de gema, altura e cor em relação ao tratamento $\mathrm{CN}$ em ovos analisados apenas no início do armazenamento. Por outro lado, utilizando rações experimentais contendo extratos de caroço e casca de manga (200 e $400 \mathrm{ppm}$ ), Freitas et al. ${ }^{(23)}$ verificaram valores de UH significativamente maiores em relação ao $\mathrm{CN}$ em ovos recém-coletados. Outros estudos ${ }^{(24)}$, suplementando a alimentação de poedeiras com dois níveis $(0,5$ e $1 \%)$ de quatro tipos de ervas (orégano, tomilho, alecrim, açafrão-da-terra) obtiveram melhoras de IG e PG em relação ao CN, mas, assim como neste estudo, não houve diferença para UH, PA e cor da gema em ovos não armazenados. Neste experimento, a inclusão dos óleos vegetais não diferiu $(\mathrm{P}<0,05)$ do $\mathrm{CN}$ para os valores de luminosidade ( $\mathrm{L}^{*}$ ) nos ovos armazenamento sob TA, contudo, ao final de 30 dias, apenas a cor das gemas com a inclusão de SC 0,03\% estavam mais claras, em comparação com inclusão de CP 0,03 e $0,06 \%$.

Nos ovos armazenados sob $\mathrm{R}$ por até 30 dias, o uso dos óleos vegetais nas dietas não afetou significativamente $(\mathrm{P}>0,05)$ os valores médios de $\mathrm{UH}, \mathrm{PC}, \mathrm{PG}, \mathrm{PA}$ e cor $\left(\mathrm{L}^{*}, \mathrm{a}^{*}, \mathrm{~b}^{*}\right)$, exceto para IG que foi significativamente melhorado $(\mathrm{P}<0,05)$ em relação ao $\mathrm{CN}$ com a inclusão de SC 0,03 e $0,06 \%$ aos 14 dias de armazenamento, porém este efeito não persistiu no decorrer do armazenamento. Dados similares foram apresentados por Liu et al. ${ }^{(17)}$ ao suplementarem a ração de poedeiras com 0,3 ou $1,0 \%$ de uma mistura de extrato de folha de amoreira, madressilva japonesa e "goldthread" e avaliarem os valores médios de $\mathrm{UH}$, altura do albúmen e cor da gema em ovos armazenados a $4{ }^{\circ} \mathrm{C}$ por duas semanas.

Ao comparar os efeitos da suplementação dos óleos vegetais nas diferentes temperaturas de armazenamento (TA x R), também não foram encontradas diferenças significativas para IG, PC, PG, PA, a* e b*, exceto para a qualidade do albúmen. Até os 7 dias de armazenamento, os valores médios de UH em TA mantiveram-se altos, exceto para CP 0,09\%. Com o aumento do tempo de armazenamento, os tratamentos CP 0,03 e $0,06 \%$ e SC $0,03 \%$ em TA mantiveram os valores médios de UH elevados $(\mathrm{p}<0,05)$, semelhantes aos tratamentos conservados sob refrigeração por 14 dias, sugerindo atividade antioxidante dos compostos bioativos presentes nos óleos.

Durante o armazenamento, $\mathrm{o} \mathrm{pH}$ do albúmen aumenta devido à perda de $\mathrm{CO}_{2}$, ocorrendo a dissociação das proteínas lisozima e ovomucina, influenciando a diminuição da viscosidade e consequente a redução de altura do albúmen ${ }^{(25,26)}$. O processo de liquefação do albúmen também pode ser explicado pela oxidação das proteínas durante o armazenamento, uma vez que a formação de peróxidos ao longo do armazenamento de alimentos, além de afetar as características sensoriais, destrói proteínas e lipídeos $^{(27)}$. Apesar das pesquisas avaliando a suplementação de poedeiras com óleos e extratos vegetais sobre os parâmetros de qualidade física ao longo do armazenamento serem escassas, alguns estudos revelam que a adição de antioxidantes naturais pode melhorar a qualidade do albúmen ${ }^{(23,28)}$. Assim, conclui-se que as menores perdas de qualidade do albúmen nos ovos dos tratamentos CP 0,03 e $0,06 \%$ e SC $0,03 \%$ armazenados em TA até 14 dias, foram decorrentes da presença de compostos 
antioxidantes nos óleos vegetais adicionados à alimentação das poedeiras.

Tabela 2. Resultados médios da qualidade física de ovos provenientes de poedeiras arraçoadas com a inclusão de óleos vegetais armazenados em diferentes temperaturas $(n=3)$

\begin{tabular}{|c|c|c|c|c|c|c|c|c|c|c|c|}
\hline Planta & Dose \% & $\mathbf{T}$ & dias & UH & IG & PC & PG & PA & $\mathbf{L}^{*}$ & $a^{*}$ & $b^{*}$ \\
\hline $\mathrm{CN}$ & 0 & TA & 0 & 87,87 & 0,49 & 9,67 & 23,33 & 67,00 & 68,06 & $-7,25$ & 40,51 \\
\hline $\mathrm{CP}$ & 0,03 & TA & 0 & 96,25 & 0,52 & 9,76 & 27,13 & 63,11 & 68,27 & $-7,06$ & 38,94 \\
\hline $\mathrm{CP}$ & 0,06 & TA & 0 & 93,68 & 0,52 & 10,43 & 25,39 & 64,21 & 70,18 & $-7,12$ & 40,89 \\
\hline $\mathrm{CP}$ & 0,09 & TA & 0 & 88,65 & 0,50 & 10,61 & 23,19 & 66,20 & 69,45 & $-6,93$ & 46,19 \\
\hline $\mathrm{SC}$ & 0,03 & TA & 0 & 88,64 & 0,50 & 10,02 & 23,38 & 66,59 & 71,12 & $-6,82$ & 45,42 \\
\hline $\mathrm{SC}$ & 0,06 & TA & 0 & 92,21 & 0,48 & 10,32 & 24,84 & 64,84 & 66,88 & $-6,58$ & 42,46 \\
\hline DP & & & & $\pm 4,87$ & $\pm 0,02$ & $\pm 0,75$ & $\pm 2,83$ & $\pm 2,84$ & $\pm 2,28$ & $\pm 0,45$ & $\pm 3,85$ \\
\hline $\mathrm{CN}$ & 0 & $\mathrm{R}$ & 7 & $94,72^{A}$ & 0,51 & 8,89 & 24,34 & 66,77 & 67,68 & $-7,05$ & 40,96 \\
\hline $\mathrm{CP}$ & 0,03 & $\mathrm{R}$ & 7 & $88,83^{A}$ & 0,51 & 10,91 & 25,67 & 63,42 & 66,34 & $-6,01$ & 44,85 \\
\hline $\mathrm{CP}$ & 0,06 & $\mathrm{R}$ & 7 & $94,50 \mathrm{~A}$ & 0,51 & 10,98 & 24,22 & 64,08 & 65,12 & $-5,41$ & 47,03 \\
\hline $\mathrm{CP}$ & 0,09 & $\mathrm{R}$ & 7 & $91,44 \mathrm{~A}$ & 0,51 & 10,44 & 24,94 & 65,34 & 65,42 & $-6,22$ & 41,64 \\
\hline $\mathrm{SC}$ & 0,03 & $\mathrm{R}$ & 7 & $86,95^{\text {B }}$ & 0,48 & 11,04 & 24,27 & 64,69 & 68,30 & $-6,68$ & 45,72 \\
\hline $\mathrm{SC}$ & 0,06 & $\mathrm{R}$ & 7 & $82,46^{\mathrm{B}}$ & 0,46 & 10,61 & 24,04 & 65,35 & 67,43 & $-6,87$ & 45,72 \\
\hline $\mathrm{CN}$ & 0 & TA & 7 & $66,16^{A}$ & 0,41 & 9,03 & 25,39 & 65,58 & 64,16 & $-6,54$ & 43,54 \\
\hline $\mathrm{CP}$ & 0,03 & TA & 7 & $77,00 \mathrm{~A}$ & 0,41 & 9,93 & 24,44 & 65,63 & 71,46 & $-6,80$ & 38,46 \\
\hline $\mathrm{CP}$ & 0,06 & TA & 7 & $77,69 \mathrm{~A}$ & 0,43 & 10,24 & 23,73 & 66,04 & 68,94 & $-6,58$ & 42,50 \\
\hline $\mathrm{CP}$ & 0,09 & TA & 7 & $55,86^{\mathrm{B}}$ & 0,4 & 10,54 & 26,52 & 62,94 & 69,34 & $-7,24$ & 46,71 \\
\hline $\mathrm{SC}$ & 0,03 & TA & 7 & $77,86^{\mathrm{A}}$ & 0,43 & 10,63 & 24,95 & 64,42 & 71,34 & $-6,29$ & 42,10 \\
\hline $\mathrm{SC}$ & 0,06 & TA & 7 & $74,03 \mathrm{~A}$ & 0,43 & 10,69 & 26,15 & 63,16 & 69,12 & $-6,60$ & 43,12 \\
\hline DP & & & & $\pm 12,36$ & $\pm 0,05$ & $\pm 0,93$ & $\pm 1,85$ & $\pm 1,93$ & $\pm 3,38$ & $\pm 0,59$ & $\pm 4,77$ \\
\hline $\mathrm{CN}$ & 0 & $\mathrm{R}$ & 14 & $81,02^{A}$ & $0,43^{b}$ & 11,09 & 23,8 & 65,11 & 66,65 & $-6,07$ & 44,42 \\
\hline $\mathrm{CP}$ & 0,03 & $\mathrm{R}$ & 14 & $89,51^{\mathrm{A}}$ & $0,53^{a b}$ & 9,80 & 23,92 & 66,28 & 68,81 & $-7,06$ & 45,43 \\
\hline $\mathrm{CP}$ & 0,06 & $\mathrm{R}$ & 14 & $78,29 \mathrm{~A}$ & $0,50^{a b}$ & 10,92 & 27,29 & 63,44 & 66,90 & $-7,08$ & 42,57 \\
\hline $\mathrm{CP}$ & 0,09 & $\mathrm{R}$ & 14 & $78,22^{A}$ & $0,51 \mathrm{ab}$ & 9,77 & 25,64 & 62,93 & 67,23 & $-6,53$ & 40,02 \\
\hline $\mathrm{SC}$ & 0,03 & $\mathrm{R}$ & 14 & $86,19 \mathrm{~A}$ & $0,52^{\mathbf{a}}$ & 9,79 & 24,47 & 65,74 & 67,47 & $-6,68$ & 46,61 \\
\hline $\mathrm{SC}$ & 0,06 & $\mathrm{R}$ & 14 & $79,60 \mathrm{~A}$ & $0,52^{\mathrm{a}}$ & 11,38 & 24,72 & 63,9 & 69,12 & $-7,09$ & 47,24 \\
\hline $\mathrm{CN}$ & 0 & TA & 14 & $27,36^{\mathrm{B}}$ & 0,34 & 10,74 & 27,95 & 61,31 & 70,22 & $-7,54$ & 44,82 \\
\hline $\mathrm{CP}$ & 0,03 & TA & 14 & $38,13^{A}$ & 0,33 & 10,82 & 25,44 & 63,74 & 73,15 & $-7,33$ & 44,28 \\
\hline $\mathrm{CP}$ & 0,06 & TA & 14 & $53,50 \mathrm{~A}$ & 0,36 & 10,47 & 24,69 & 64,84 & 70,32 & $-7,19$ & 52,30 \\
\hline $\mathrm{CP}$ & 0,09 & TA & 14 & $23,01^{\mathrm{B}}$ & 0,32 & 11,63 & 28,04 & 60,33 & 66,6 & $-6,81$ & 52,55 \\
\hline $\mathrm{SC}$ & 0,03 & TA & 14 & $42,04 \mathrm{~A}$ & 0,32 & 11,88 & 29,07 & 59,06 & 67,32 & $-7,16$ & 43,72 \\
\hline $\mathrm{SC}$ & 0,06 & TA & 14 & $35,24^{\mathrm{B}}$ & 0,34 & 11,39 & 27,27 & 61,34 & 72,39 & $-7,28$ & 49,75 \\
\hline DP & & & & $\pm 28,98$ & $\pm 0,09$ & $\pm 1,23$ & $\pm 2,47$ & $\pm 3,26$ & $\pm 4,02$ & $\pm 0,66$ & $\pm 4,69$ \\
\hline $\mathrm{CN}$ & 0 & $\mathrm{R}$ & 21 & $98,35^{\mathrm{A}}$ & 0,53 & 10,18 & 24,76 & 65,06 & 69,33 & $-6,83$ & 49,16 \\
\hline $\mathrm{CP}$ & 0,03 & $\mathrm{R}$ & 21 & $80,13^{\text {B }}$ & 0,53 & 10,39 & 26,88 & 62,73 & 63,63 & $-6,76$ & 43,66 \\
\hline $\mathrm{CP}$ & 0,06 & $\mathrm{R}$ & 21 & $80,35^{\text {B }}$ & 0,51 & 10,06 & 24,16 & 60,24 & 67,61 & $-7,00$ & 47,78 \\
\hline $\mathrm{CP}$ & 0,09 & $\mathrm{R}$ & 21 & $80,22^{\text {B }}$ & 0,49 & 11,03 & 30,30 & 64,81 & 66,93 & $-6,86$ & 48,32 \\
\hline $\mathrm{SC}$ & 0,03 & $\mathrm{R}$ & 21 & $84,68 \mathrm{~A}$ & 0,52 & 10,10 & 29,74 & 60,70 & 66,46 & $-6,24$ & 49,37 \\
\hline $\mathrm{SC}$ & 0,06 & $\mathrm{R}$ & 21 & $79,59^{\text {В }}$ & 0,49 & 11,18 & 23,78 & 65,04 & 64,76 & $-6,60$ & 48,99 \\
\hline $\mathrm{CN}$ & 0 & TA & 21 & $37,66^{\mathrm{B}}$ & 0,33 & 10,76 & 25,78 & 63,46 & 68,17 & $-6,61$ & 52,10 \\
\hline $\mathrm{CP}$ & 0,03 & TA & 21 & $24,80^{\mathrm{C}}$ & 0,31 & 10,57 & 26,15 & 63,27 & 64,39 & $-6,57$ & 52,84 \\
\hline
\end{tabular}


Continuacão...

\begin{tabular}{|c|c|c|c|c|c|c|c|c|c|c|c|}
\hline Planta & Dose \% & $\mathbf{T}$ & dias & UH & IG & PC & PG & PA & $\mathbf{L}^{*}$ & $a^{\star}$ & $\mathbf{b}^{\star}$ \\
\hline $\mathrm{CP}$ & 0,06 & $\mathrm{TA}$ & 21 & $32,93^{\mathrm{C}}$ & 0,32 & 10,26 & 26,22 & 63,53 & 70,80 & $-6,45$ & 50,32 \\
\hline $\mathrm{CP}$ & 0,09 & TA & 21 & $20,76^{\mathrm{C}}$ & 0,30 & 10,72 & 28,54 & 60,74 & 68,71 & $-7,1$ & 8 \\
\hline $\mathrm{SC}$ & 0,03 & TA & 21 & $28,99 \mathrm{C}$ & 0,27 & 12,20 & 27,20 & 60 & 53,64 & -5 & 49, \\
\hline $\mathrm{SC}$ & 0,06 & TA & 21 & $28,27^{\mathrm{C}}$ & 0,30 & 10,99 & 26,81 & 62,2 & 64, & & \\
\hline DP & & & & $\pm 30,69$ & $\pm 0,11$ & & $\pm 2,78$ & & $\pm 5,12$ & & \\
\hline $\mathrm{CN}$ & 0 & $\mathrm{R}$ & 30 & $83,71^{A}$ & 0,53 & 10,78 & 27,66 & 61,56 & $65,17^{\mathrm{B}}$ & $-6,07$ & 43,28 \\
\hline $\mathrm{CP}$ & & $\mathrm{R}$ & 30 & $81,83 \mathrm{~A}$ & & 10,86 & 27,25 & & $67,34 \mathrm{AB}$ & & \\
\hline $\mathrm{CP}$ & & $\mathrm{R}$ & 30 & $78,40^{\mathrm{C}}$ & 0,55 & 10,82 & 31,29 & & 67 & & 52,01 \\
\hline $\mathrm{CP}$ & 09 & $\mathrm{R}$ & 30 & $78,44^{\text {B }}$ & 0,51 & 10,64 & 24,03 & 58, & $68,91 \mathrm{AB}$ & -7 & 44,36 \\
\hline $\mathrm{SC}$ & & $\mathrm{R}$ & 30 & $81,29 \mathrm{~A}$ & 0,53 & & 26 , & & 68 & & 50, \\
\hline $\mathrm{SC}$ & 0,06 & $\mathrm{R}$ & 30 & $81,35^{A}$ & 0,52 & 10,25 & 25,99 & & $69,71 \mathrm{AB}$ & -7 , & 51,96 \\
\hline $\mathrm{CN}$ & 0 & TA & 30 & $19,88^{\mathrm{D}}$ & 0,33 & 10,55 & 27,86 & & $71,98^{\mathrm{Bab}}$ & -6 , & 52,7 \\
\hline $\mathrm{CP}$ & 0,03 & TA & 30 & $27,32^{\mathrm{B}}$ & 0,28 & 10,67 & 28,46 & 60 , & $69,73 \mathrm{Bb}$ & -6 , & 49 \\
\hline $\mathrm{CP}$ & & TA & 30 & $28,56^{\mathrm{D}}$ & 0,31 & 10 & 28,46 & 60 , & $68,48^{\mathrm{Bb}}$ & & 8 \\
\hline $\mathrm{CP}$ & & TA & 30 & $31,89^{\mathrm{B}}$ & & & 30 & & $4^{\mathrm{Bab}}$ & & \\
\hline $\mathrm{SC}$ & 0,03 & TA & 30 & $26,47^{\mathrm{D}}$ & 0,3 & & 29 , & & $5 \mathrm{Aa}$ & -7 & 7 \\
\hline $\mathrm{SC}$ & 0,06 & TA & 30 & $41,32^{\mathrm{BC}}$ & 0,3 & & 26,15 & & $72,14^{\mathrm{Bab}}$ & & \\
\hline $\mathrm{DP}$ & & & & $\pm 28,21$ & $\pm 0,11$ & $\pm 0,79$ & $\pm 2,46$ & & $\pm 3,59$ & $\pm 0,74$ & $\pm 5,84$ \\
\hline \multicolumn{4}{|c|}{$\begin{array}{l}\text { P-valor (dia } \mathrm{x} \text { planta } \mathrm{x} \\
\text { níveis } \mathrm{x} T \text { ) }\end{array}$} & $<0,0001$ & 0,0997 & 0,3217 & 0,0067 & 0,0028 & 0,0008 & 0,0233 & $<0,0001$ \\
\hline \multicolumn{12}{|c|}{$\begin{array}{l}\text { Médias seguidas de letras minúsculas na mesma coluna diferem estatisticamente na mesma temperatura de } \\
\text { armazenamento pelo Teste de Tukey }(\mathrm{P}<0,05) ; \mathrm{e}^{2} \mathrm{Letras} \text { maiúsculas na mesma coluna diferem estatisticamente entre os } \\
\text { tratamentos nas diferentes temperaturas de armazenamento }(\mathrm{RxTA}) \text {. } \mathrm{DP}=\text { desvio padrão; } \mathrm{CN}=\text { controle negativo; } \mathrm{CP}= \\
\text { copaíba; } \mathrm{SC}=\text { sucupira; } \mathrm{T}=\text { temperatura de armazenamento; } \mathrm{R}=\text { refrigeração; } \mathrm{TA}=\text { temperatura ambiente; } \mathrm{UH}= \\
\text { Unidade Haugh; } \mathrm{IG}=\text { indice de gema; } \mathrm{PC}=\text { porcentagem de casca; } \mathrm{PG}=\text { porcentagem de gema; } \mathrm{PA}=\text { porcentagem de } \\
\text { albúmen; Cor }\left(\mathrm{L}^{*}, \mathrm{a}^{*}, \mathrm{~b}^{*}\right) \text {. }\end{array}$} \\
\hline
\end{tabular}

Aos 21 dias de armazenamento, a adição dos óleos vegetais não foi eficiente em preservar a qualidade dos ovos armazenados em TA, uma vez que estes apresentaram valores médios de UH significativamente inferiores $(\mathrm{P}<0,05)$ ao $\mathrm{CN}$ sob $\mathrm{R}$. Após 30 dias de armazenamento em $\mathrm{TA}$, todos os tratamentos apresentaram valores médios de UH significativamente inferiores $(\mathrm{P}<0,05)$ ao $\mathrm{CN}$ sob $\mathrm{R}$, inclusive os tratamentos com a adição dos óleos vegetais. No entanto, apesar de ser esperado que todos os ovos após 30 dias de armazenamento em TA diferissem dos ovos refrigerados, a adição de CP $0,03 \%$ e $0,09 \%$ e de SC 0,06\% em TA apresentou valores médios de UH semelhantes aos CP 0,06 e $0,09 \%$ sob R, o que sugere alguma atividade antioxidante dos óleos vegetais na preservação da qualidade do albúmen. Barbosa et al. ${ }^{(29)}$ relatam a ocorrência de um decréscimo linear nos valores de UH em ovos armazenados em TA em relação aos submetidos a refrigeração, uma vez que baixas temperaturas tendem a manter uma melhor qualidade de albúmen por até 35 dias em ovos comerciais, sem aditivos com bioativos antioxidantes.

A adição dos óleos não influenciou a coloração das gemas dos ovos, exceto SC 0,03\% em TA, que resultou em maiores $(\mathrm{P}<0,05)$ valores de $\mathrm{L}^{*}$, indicando gemas mais claras que o $\mathrm{CN}$ refrigerado. $\mathrm{O}$ índice de coloração das gemas em ovos in natura pode apresentar-se mais claro tanto em ovos armazenados em TA quanto sob R, sendo maiores em temperatura ambiente ${ }^{(30)}$.

Ao prolongar o armazenamento refrigerado por até 60 dias (Tabela 3), os valores obtidos para IG e UH foram elevados durante todo o período experimental, independente do tratamento aplicado. Sendo a refrigeração um fator decisivo na preservação da qualidade interna ${ }^{(31)}$, conclui-se que o bom estado de qualidade interna dos ovos foi decorrente do armazenamento refrigerado. Também não houve 
diferença $(\mathrm{P}>0,05)$ nos valores de $\mathrm{PC}, \mathrm{PG}, \mathrm{PA}$ e cor entre os tratamentos aplicados até o final do armazenamento refrigerado. No entanto, foi observado uma redução $(\mathrm{P}<0,05)$ do valor médio de IG no tratamento SC $0,06 \%$ aos 35 dias em relação ao CP 0,03\%, mas não houve diferença em relação ao $\mathrm{CN}$ ou demais tratamentos, nem persistência durante o período de armazenamento. Siebel e SouzaSoares ${ }^{(32)}$ explicam que a redução do IG ocorre devido ao movimento de água do albúmen para a gema, ocasionando o alargamento e redução da altura da mesma.

Tabela 3. Resultados médios da qualidade física de ovos provenientes de poedeiras arraçoadas com a inclusão de óleos vegetais armazenados sob refrigeração a $4^{\circ} \mathrm{C}(\mathrm{n}=3)$

\begin{tabular}{|c|c|c|c|c|c|c|c|c|c|c|}
\hline Planta & $\begin{array}{c}\text { Níveis } \\
\%\end{array}$ & dias & UH & IG & PC & PG & PA & $\mathbf{L}^{*}$ & $a^{*}$ & $\mathbf{b}^{*}$ \\
\hline $\mathrm{CN}$ & 0 & 35 & 81,62 & 0,51 ab & 10,45 & 26,76 & 62,79 & 66,73 & $-6,93$ & 46,31 \\
\hline $\mathrm{CP}$ & 0,03 & 35 & 79,28 & $0,55^{\mathrm{a}}$ & 10,82 & 25,45 & 63,73 & 66,32 & $-6,63$ & 45,49 \\
\hline $\mathrm{CP}$ & 0,06 & 35 & 80,49 & $0,49 a b$ & 11,19 & 25,51 & 63,3 & 65,37 & $-6,27$ & 49,18 \\
\hline $\mathrm{CP}$ & 0,09 & 35 & 71,30 & 0,52 ab & 11,18 & 25,19 & 63,63 & 66,18 & $-6,66$ & 49,84 \\
\hline $\mathrm{SC}$ & 0,03 & 35 & 84,36 & $0,53 \mathrm{ab}$ & 11,75 & 25,69 & 62,99 & 65,93 & $-6,69$ & 48,09 \\
\hline $\mathrm{SC}$ & 0,06 & 35 & 70,42 & $0,46^{b}$ & 11,35 & 25,67 & 61,56 & 63,38 & $-5,84$ & 49,66 \\
\hline DP & & & $\pm 6,74$ & $\pm 0,04$ & $\pm 0,73$ & $\pm 1,26$ & $\pm 1,45$ & $\pm 2,82$ & $\pm 0,61$ & $\pm 4,01$ \\
\hline $\mathrm{CN}$ & 0 & 42 & 78,54 & 0,52 & 10,23 & 28,21 & 61,56 & 65,14 & $-6,93$ & 45,00 \\
\hline $\mathrm{CP}$ & 0,03 & 42 & 75,03 & 0,51 & 10,92 & 26,86 & 62,22 & 65,44 & $-6,46$ & 46,06 \\
\hline $\mathrm{CP}$ & 0,06 & 42 & 77,49 & 0,51 & 10,70 & 25,37 & 63,93 & 33,24 & $-6,02$ & 49,88 \\
\hline $\mathrm{CP}$ & 0,09 & 42 & 79,35 & 0,51 & 10,85 & 27,59 & 61,57 & 67,02 & $-6,45$ & 53,82 \\
\hline $\mathrm{SC}$ & 0,03 & 42 & 80,00 & 0,51 & 10,22 & 24,33 & 65,45 & 67,38 & $-6,67$ & 50,80 \\
\hline $\mathrm{SC}$ & 0,06 & 42 & 81,11 & 0,52 & 10,63 & 25,72 & 63,65 & 67,16 & $-6,22$ & 49,16 \\
\hline DP & & & $\pm 4,26$ & $\pm 0,02$ & $\pm 1,11$ & $\pm 1,84$ & $\pm 2,14$ & $\pm 1,90$ & $\pm 0,59$ & $\pm 4,36$ \\
\hline $\mathrm{CN}$ & 0 & 49 & 73,27 & 0,49 & 10,03 & 26,46 & 63,51 & 66,44 & $-6,91$ & 47,47 \\
\hline $\mathrm{CP}$ & 0,03 & 49 & 74,46 & 0,50 & 11,83 & 27,55 & 60,62 & 66,96 & $-6,43$ & 50,78 \\
\hline $\mathrm{CP}$ & 0,06 & 49 & 74,25 & 0,51 & 11,18 & 25,79 & 63,02 & 67,67 & $-6,38$ & 52,67 \\
\hline $\mathrm{CP}$ & 0,09 & 49 & 76,47 & 0,49 & 9,96 & 26,17 & 63,87 & 64,09 & $-7,26$ & 42,95 \\
\hline $\mathrm{SC}$ & 0,03 & 49 & 75,79 & 0,51 & 11,12 & 25,68 & 63,20 & 65,74 & $-6,77$ & 44,74 \\
\hline $\mathrm{SC}$ & 0,06 & 49 & 78,94 & 0,53 & 11,02 & 26,10 & 62,88 & 65,41 & $-6,11$ & 50,18 \\
\hline DP & & & $\pm 5,33$ & $\pm 0,02$ & $\pm 1,01$ & $\pm 2,19$ & $\pm 2,39$ & $\pm 2,20$ & $\pm 0,62$ & $\pm 4,34$ \\
\hline $\mathrm{CN}$ & 0 & 60 & 73,75 & 0,49 & 10,75 & 27,15 & 62,10 & 65,53 & $-6,17$ & 44,05 \\
\hline $\mathrm{CP}$ & 0,03 & 60 & 73,04 & 0,50 & 10,51 & 28,63 & 60,86 & 61,93 & $-6,24$ & 45,10 \\
\hline $\mathrm{CP}$ & 0,06 & 60 & 71,36 & 0,47 & 11,05 & 27,26 & 61,70 & 64,98 & $-6,15$ & 50,77 \\
\hline $\mathrm{CP}$ & 0,09 & 60 & 72,56 & 0,49 & 10,85 & 26,05 & 63,09 & 66,00 & $-6,77$ & 50,86 \\
\hline $\mathrm{SC}$ & 0,03 & 60 & 71,01 & 0,47 & 11,15 & 26,78 & 62,07 & 67,18 & $-6,79$ & 50,54 \\
\hline $\mathrm{SC}$ & 0,06 & 60 & 77,07 & 0,48 & 10,17 & 27,82 & 62,01 & 65,13 & $-6,89$ & 49,48 \\
\hline DP & & & $\pm 4,65$ & $\pm 0,02$ & $\pm 0,85$ & $\pm 1,37$ & $\pm 1,47$ & $\pm 2,49$ & $\pm 0,53$ & $\pm 4,98$ \\
\hline $\begin{array}{l}P \text {-valor } \\
\text { niveis) }\end{array}$ & & & $<0,0001$ & 0,0011 & 0,5165 & 0,1291 & 0,2400 & 0,3814 & 0,3512 & 0,0529 \\
\hline
\end{tabular}

Médias seguidas de letras minúsculas na mesma coluna diferem estatisticamente entre os tratamentos pelo Teste de Tukey $(\mathrm{P} \in 0,05) . \mathrm{DP}=$ destio padrão; $\mathrm{CN}=$ controle negativo; $\mathrm{CP}=$ copaiba; $\mathrm{SC}=$ sucupira; $\mathrm{UH}=$ Unidade Haugh; IG $=$ índice de gema; $\mathrm{PC}=$ porcentagem de casca; $\mathrm{PG}=$ porcentagem de gema; $\mathrm{PA}=$ porcentagem de albúmen; $\mathrm{Cor}\left(\mathrm{L}^{*}\right.$, $\left.a^{*}, b^{*}\right)$.

A suplementação das dietas das poedeiras com os óleos de sucupira e copaíba não influenciou $(\mathrm{P}<0,05)$ os valores médios do $\mathrm{pH}$ do albúmen ou da gema (Tabela 4) dos ovos armazenados em temperatura ambiente ou sob refrigeração. Diferente desse estudo ${ }^{(33)}$, a utilização de $400 \mathrm{mg}$ de licopeno como antioxidante natural na ração de poedeiras resultou em maiores valores de $\mathrm{pH}$ iniciais do albúmen e da gema em relação ao tratamento controle em ovos armazenados sob refrigeração no 
dia zero, mas não houve efeito significativo aos 30 dias para o $\mathrm{pH}$ da gema.

Quando comparados os dados entre as diferentes temperaturas (R x TA), os ovos do tratamento CN mantidos em TA apresentaram valores médios de $\mathrm{pH}$ da gema superiores $(\mathrm{P}<0,05)$ ao $\mathrm{CN}$ sob $\mathrm{R}$, mas não diferiram $(\mathrm{P}<0,05)$ dos tratamentos com inclusão de $\mathrm{CP}$ em ovos refrigerados.

Por outro lado, a adição de óleos vegetais nas dietas de poedeiras resultou em valores de $\mathrm{pH}$ da gema em TA semelhantes $(\mathrm{P}>0,05)$ aos dos ovos sob $\mathrm{R}$, sugerindo que a presença dos antioxidantes naturais provocou efeito positivo na integridade da membrana da gema e, consequentemente, na estabilidade do $\mathrm{pH}$ da gema em TA por até 30 dias. Shang et al. ${ }^{(34)}$ relacionam a elevação de $\mathrm{pH}$ das gemas ao aumento da permeabilidade da membrana vitelina durante o armazenamento, favorecendo a difusão dos íons $\mathrm{H}^{+}$da gema para o albúmen e então, deixando o $\mathrm{pH}$ mais alcalino. A deterioração da membrana vitelina ao longo do armazenamento decorre do rompimento das ligações cruzadas das proteínas que compõem sua estrutura ${ }^{(35,36)}$ e, sabendo-se que as proteínas e lipídeos de ovos sofrem oxidação ao longo do tempo ${ }^{(27)}$, sugere-se que os antioxidantes presentes nos óleos vegetais tenham retardado esse processo.

Tabela 4. Valores médios do $\mathrm{pH}$ da gemas e albúmen de ovos provenientes de poedeiras arraçoadas com a inclusão de óleos vegetais, armazenados em diferentes temperaturas ( $n=3$ )

\begin{tabular}{|c|c|c|c|c|c|c|c|c|c|c|}
\hline \multicolumn{2}{|l|}{ Gema } & \multicolumn{4}{|c|}{$\begin{array}{l}\text { Periodo de armazenamento } \\
\text { (dias) }\end{array}$} & \multirow[b]{2}{*}{30} & \multirow[b]{2}{*}{35} & \multirow[b]{2}{*}{42} & \multirow[b]{2}{*}{49} & \multirow[b]{2}{*}{60} \\
\hline Planta & $\mathrm{T}$ & 0 & 7 & 14 & 21 & & & & & \\
\hline $\mathrm{CN}$ & $\mathrm{R}$ & 5,91 & 5,97 & 5,92 & 6,14 & $6,10^{\mathrm{B}}$ & 6,22 & 6,39 & 6,32 & 6,52 \\
\hline $\mathrm{CP}$ & $\mathrm{R}$ & 5,91 & 5,98 & 6,16 & 6,13 & $6,33 \mathrm{~A}$ & 6,22 & 6,29 & 6,08 & 6,14 \\
\hline $\mathrm{SC}$ & $\mathrm{R}$ & 6,01 & 6,03 & 6,08 & 6,28 & $6,22^{\mathrm{B}}$ & 6,11 & 6,36 & 6,14 & 6,22 \\
\hline $\mathrm{CN}$ & TA & 5,91 & 6,14 & 6,33 & 6,29 & $6,86^{A}$ & $*$ & $*$ & $*$ & $*$ \\
\hline $\mathrm{CP}$ & TA & 5,91 & 5,95 & 6,16 & 6,14 & $6,33 A B$ & * & $*$ & $*$ & $*$ \\
\hline $\mathrm{SC}$ & $\mathrm{TA}$ & 6,01 & 6,02 & 6,05 & 6,16 & $6,43 A B$ & $*$ & $*$ & $*$ & $*$ \\
\hline $\mathrm{DP}$ & & $\pm 0,06$ & $\pm 0,07$ & $\pm 0,17$ & $\pm 0,09$ & $\pm 0,23$ & $\pm 0,06$ & $\pm 0,08$ & $\pm 0,16$ & $\pm 0,20$ \\
\hline \multicolumn{11}{|l|}{ Albúmen } \\
\hline $\mathrm{CN}$ & $\mathrm{R}$ & 8,33 & 8,93 & 9,14 & 9,26 & 9,33 & 9,22 & 9,21 & 8,99 & 9,02 \\
\hline $\mathrm{CP}$ & $\mathrm{R}$ & 8,47 & 9,01 & 9,28 & 9,26 & 9,25 & 9,27 & 9,24 & 9,02 & 9,10 \\
\hline $\mathrm{SC}$ & $\mathrm{R}$ & 8,51 & 9,06 & 9,19 & 9,24 & 9,25 & 9,23 & 9,19 & 9,05 & 9,51 \\
\hline $\mathrm{CN}$ & TA & 8,33 & 9,26 & 9,44 & 9,56 & 9,49 & $*$ & $*$ & $*$ & $*$ \\
\hline $\mathrm{CP}$ & $\mathrm{TA}$ & 8,47 & 9,41 & 9,55 & 9,64 & 9,50 & $*$ & $*$ & $*$ & $*$ \\
\hline $\mathrm{SC}$ & TA & 8,51 & 9,41 & 9,52 & 9,60 & 9,48 & $*$ & $*$ & $*$ & $*$ \\
\hline DP & & $\pm 0,19$ & $\pm 0,21$ & $\pm 0,17$ & $\pm 0,19$ & $\pm 0,13$ & $\pm 0,03$ & $\pm 0,05$ & $\pm 0,07$ & $\pm 0,37$ \\
\hline$P$ - valor & & \multicolumn{4}{|c|}{ pH (gema) } & \multicolumn{2}{|c|}{ pH (albúmen) } & & & \\
\hline Dia x planta $\mathrm{x}$ & & & 0,0002 & & & $<0,0001$ & & & & \\
\hline \multicolumn{11}{|l|}{$\mathrm{T}(\mathrm{R} \times \mathrm{TA})$} \\
\hline \multicolumn{2}{|c|}{ Dia x planta x $T(R)$} & \multicolumn{3}{|c|}{0,0070} & & \multicolumn{2}{|c|}{$<0,0001$} & & & \\
\hline
\end{tabular}

'Médias seguidas de letras minúsculas diferentes na mesma coluna diferem estatisticamente na mesma temperatura de armazenamento pelo Teste de Tukey $(\mathrm{P}<0,05)$; e ${ }^{2}$ Letras maiúsculas diferentes na mesma coluna diferem estatisticamente entre os tratamentos nas diferentes temperaturas de armazenamento. *Não analisado; $\mathrm{DP}=$ desvio padrão; $\mathrm{CN}=$ controle negativo; $\mathrm{CP}=$ copaiba; $\mathrm{SC}=$ sucupira; $\mathrm{R}=$ refrigeração; $\mathrm{TA}=$ temperatura ambiente; $\mathrm{T}=$ temperatura de armazenamento. 


\section{Conclusão}

A suplementação dos óleos vegetais de copaíba $(0,03$ e $0,06 \%)$ e sucupira $(0,03 \%)$ nas dietas de poedeiras pode auxiliar na preservação da qualidade do albúmen dos ovos mantidos em temperatura ambiente por até 14 dias, de forma semelhante ao uso da refrigeração. Pode ainda auxiliar a manutenção do $\mathrm{pH}$ das gemas em ovos armazenados em temperatura ambiente por até 30 dias. Porém, o uso dos óleos vegetais nas dietas de poedeiras não melhorou a qualidade interna dos ovos armazenados sob refrigeração, sob nenhum aspecto estudado.

\section{Agradecimentos}

Este estudo teve o apoio do Decanato de Pós-Graduação da Universidade de Brasília (DPP/UnB), do CNPq através do Projeto Produção Animal Sustentável (PAS) da Rede Multidisciplinar Pró-CentroOeste e o apoio financeiro de bolsa da Fundação Coordenação de Aperfeiçoamento de Pessoal de Nível Superior (CAPES).

\section{Referências}

1. Bessa NGF, Borges, JCM, Beserra, FP, Carvalho RHA, Pereira, MAB, Fagundes R, Campos SL, Ribeiro LU, Quirino MS, Chagas Junior AF, Alves A. Prospecção fitoquímica preliminar de plantas nativas do cerrado de uso popular medicinal pela comunidade rural do assentamento vale verde - Tocantins. Revista Brasileira de Plantas Medicinais. 2013; 15(4): 692-707.

2. Melo PS, Bergamaschi KB, Tiveron AP, Massarioli AP, Oldoni TLC, Zanus MC, Pereira GE, Alencar SM. Composição fenólica e atividade antioxidante de resíduos agroindustriais. Ciência Rural. 2011; 41(6):10881093.

3. Montes LV, Broseghini LP, Andreata FS, Sant'Anna MESNeves VM, Silva AG. Evidências para o uso da óleo-resina de copaíba na cicatrização de ferida - uma revisão sistemática. Natureza on line. 2009 [citado em 24 de maio de 2016]; 7(2):61-67. Disponível de http://www.naturezaonline.com.br/natureza/conteudo/pdf/02_monteslvetal 6167.pdf.

4. Muniz JWC, Bozza PT, Nascimento JLM, Reis PA. Atividade anti-inflamatória do óleo-resina da Copaifera reticulata em modelo inflamatório de edema de pata. Revista Paraense de Medicina. 2010; 23(1):2031-2037.

5. Mendonça DE, Onofre SB. Atividade antimicrobiana do óleo-resina produzido pela copaíba - Copaifera multijuga Haine (Leguminosae). Revista Brasileira de Farmacognosia. 2009; 29(2B):577-581.

6. Pieri FA, Mussi MC, Moreira MAS. Óleo de copaíba (Copaifera sp.): histórico, extração, aplicações industriais e propriedades medicinais. Revista Brasileira de Plantas Medicinais. 2009; 11(4):465-472.

7. Oliveira DMT, Paiva EAS. Anatomy and Ontogeny of Pterodon emarginatus (FABACEAE: FABOIDEAE) Seed. Brazilian Journal of Pharmaceutical Sciences. 2005; 65(3): p.483-494.

8. Dutra RC, Fava MB, Alves CC, Ferreira AP, Raposo NRB. Antiulcerogenic and anti-inflammatory activities of the essential oil from Pterodon emarginatus seeds. Journal of Pharmacy and Pharmacology. 2009; 61(2):243-250.

9. Bustamante KGL, Lima ADF, Soares ML, Fiuza TS, Tresvenzol LMF, Bara MTF, Pimenta F, Paula JR. Avaliação da atividade antimicrobiana do extrato etanólico bruto da casca da sucupira branca (Pterodon emarginatus Vogel) - Fabaceae. Revista Brasileira de Plantas Medicinais. 2010; 12(3):341-345. 
10. Mors WB, Santos FMF, Monteiro HJ, Gilber B. Chemoprophylatic agent in schistossomiasis: 14,15epoxygeranilgeraniol. Science. 1967; 157: 950-951.

11. Dutra RC, Leite MN, Barbosa N R. Quantification of Phenolic Constituents and Antioxidant Activity of Pterodon emarginatus Vogel Seeds. International Journal of Molecular Sciences. 2008; 9(4):606-614.

12. Donati M, Mondin A, Chen Z, Miranda FM, Nascimento BB Jr, Schirato G, Pastore P, Froldi G. Radical scavenging and antimicrobial activities of Croton zehntneri, Pterodon emarginatus and Schinopsis brasiliensis essential oils and their major constituents: estragole, trans-anethole, $\beta$-caryophyllene and myrcene. Natural Product research. 2015; 29(10):939-946.

13. Silva FAM, Borges MFM, Ferreira MA. Métodos para avaliação do grau de oxidação lipídica e da capacidade antioxidante. Química Nova. 1999; 22(1):94-103.

14. Cherian G, Wolfe EH, Sim JS. Feeding dietary oil with tocopherols: effect of internal qualities of eggs during storage. Journal of Food Science. 1996; 61(1):15-18.

15. Hayat Z, Cherian G, Pasha TN, Khattak FM, Jabbar MA. Oxidative stability and lipid components of eggs from flax-fed hens: Effect of dietary antioxidants and storage. Poultry Science. 2010; 89(6):1285-1292.

16. Garcia ERM, Orlandi CCB, Oliveira CAL, Cruz FK, Santos TMB, Otutumi LK. Qualidade de ovos de poedeiras semipesadas armazenados em diferentes temperaturas e períodos de estocagem. Revista Brasileira de Saúde e Produção Animal. 2010; 11(2):505-518.

17. Liu XD, Jang A, Lee BD, Lee M, Jo C. Effect of Dietary Inclusion of Medicinal Herb Extract Mix in a Poultry Ration on the Physico-chemical Quality and Oxidative Stability of Eggs. Journal of Animal Science. 2009; 22(3):421-427.

18. Florou-Paneri P, Nikolakakis I, Giannenas A, Koidis A, Botsoglou E, Dotas V, Mitsopoulos I. Hen Performance and Egg Quality as Affected by Dietary Oregano Essential Oil and Alpha-tocopheryl Acetate Supplementation. Journal of Poultry Science. 2005; 4(7):449-454.

19. Pascoal LAF, Bento BA Junior, Santos WS, Silva LS, Dourado LRB, Bezerra ABA. Qualidade dos ovos comercializados em diferentes estabelecimentos na cidade de Imperatriz-MA. Revista Brasileira de Saúde e Produção Animal. 2008; 9(1):150-157.

20. Brasil. Ministério da Agricultura, Pecuária e Abastecimento. Portaria n. ${ }^{\circ} 01$ de 21 de fevereiro de 1990. Resolvem aprovar Normas Gerais de Inspeção de Ovos e Derivados, propostas pela Divisão de inspeção de Carnes e Derivados - DICAR. Diário Oficial da União. 1990 Mar 06; Seção 1. Portuguese.

21. Rostagno HS, Albino LFT, Donzele JL, Gomes PC, Oliveira RF, Lopes DC, Ferreira AS, Barreto SLT, Euclides RF. Tabelas brasileiras para aves e suínos: composição de alimentos e exigências nutricionais. $3^{\mathrm{a}}$ ed. Viçosa, MG: Universidade Federal de Viçosa; 2011. 252p.

22. SAS, Institute. Statistical Analysis System: user guide [CD-ROM]. Version 9.3. Cary (NC): SAS Insitute Inc., 2013.

23. Freitas ER, Borges AS, Trevisan MTS, Cunha AL, Braz NM, Watanabe PH, Nascimento GAJ. Extratos etanólicos de manga como antioxidantes na alimentação de poedeiras. Pesquisa Agropecuária Brasileira. 2013; 48(7):714-721.

24. Radwan NL, Hassan RA, Qota EM, Fayek HM. Effect of Natural Antioxidant on Oxidative Stability of Eggs and Productive and Reproductive Performance of Laying Hens. International Journal of Poultry Science. 2008; 7(2):134-150.

25. Lucisano M, Hidalgo A, Comelli EM, Rossi M. Evalution of chemical and physical albumen characteristics during the storage of shell eggs. Journal of Agricultural and Food Chemistry. 1996; 44(5):1235-1240.

26. Kemps BJ, Bamelis FR, Mertens K, Decuypere EM, De Baerdemaeker JG, De Ketelaere B. The assessment of viscosity measurements on the albumen of consumption eggs as an indicator for freshness. Poultry Science. 
2010; 89(12):2699-2703.

27. Mehdizadeh SA, Minaei S, Torshizi MAK, Mohajerani E. Effect of UV irradiation, sample thickness and storage temperature on storability, bacterial activity and functional properties of liquid egg. Journal of Food Science and Technology. 2015; 52(7):4414-4422.

28. Özek K, Wellmann KT, Ertekin B, Tarim B. Effects of dietary herbal essential oil mixture and organic acid preparation on laying traits, gastrointestinal tract characteristics, blood parameters and immune response of laying hens in a hot summer season. Journal of Animal and Feed Sciences. 2011; 21(4):575-586.

29. Barbosa N AA, Sakomura NK, Mendonça MO, Freitas ER, Fernandes JBK. Qualidade de ovos comerciais provenientes de poedeiras Comerciais armazenados sob diferentes tempos e condições de ambientes. Ars Veterinaria. 2008; 24(2):127-133.

30. Santos MSV, Espíndola GB, Lôbo RNB, Freitas ER, Guerra JLL, Santos ABE. Efeito da temperatura e estocagem em ovos. Ciência e Tecnologia de Alimentos. 2009; 29(3):513-517.

31. Jucá TS, Gomes FA, Silva LA, Silva RPM, Vale MAD. Efeito do tempo e condições de armazenamento sobre a qualidade interna de ovos de poedeiras isa brown produzidos em diferentes sistemas de criação e ambiência. Enciclopédia Biosfera, Centro Científico Conhecer. 2011; 7(13):446.

32. Siebel NF, Souza-Soares LA. Avaliação física de ovos de codornas em diferentes períodos de armazenamento. Vetor. 2003; 13:47-52.

33. Cruz FK, Garcia ERM, Ferraz ALJ, Souza KMR, Feliciano WB, Rohod RV. Quality and stability of eggs from laying hens fed with organic minerals and lycopene. Ciência Rural. 2016; 46(1):157-162.

34. Shang XG, Wang FL, Li DF, Yin DJ, Li JY. Effects of dietary conjugated linoleic acid on the productivity of laying hens and egg quality during refrigerated storage. Poultry Science. 2004; 83(10):16881695 .

35. Mineki M, Kobayashi M. Microstructure changes in stored hen egg yolk. Japan Poultry Science Association. 1998; 35(5):285-294.

36. Fromm D. Some physical changes in the perivitelline layer of the hen's egg during storage. Journal of Food Science. 1967; 32: 52-56. 